www.jkns.or.kr

Case Report

Jong-Eun Sim, M.D.

Seung-Jin Noh, M.D.

Young-Jin Song, M.D.

Hyung-Dong Kim, M.D.

Department of Neurosurgery

College of Medicine

Dong-A University

Busan, Korea

\section{Removal of Intradural-Extramedullary Spinal Cord Tumors with Unilateral Limited Laminectomy}

Total laminectomy for the removal of intradural-extramedullary spinal cord tumors has been used widely, but postoperative complications often develop, such as kyphosis, spinal instability, and persistent back pain. In this study, we evaluated seven patients with intradural-extramedullary spinal cord tumors with respect to the value of unilateral limited laminectomy. Our cases included six schwannomas, and one meningioma. The cervical region was involved in four cases, the thoracolumbar region in two cases, and the lumbar region in one case. The rationale for choosing a unilateral approach is to preserve musculoligamentous attachments and posterior bony elements as much as possible. The patients were mobilized on the third postoperative day and preoperative neurological symptoms were recovered within a few weeks. We did not observe any complication relating to unilateral limited laminectomy and at follow-up evaluation (at 3 and 12 months postoperatively), none of the patients showed spinal deformity or spinal instability. We think that the unilateral limited laminectomy is a safe and efficient technique for the treatment of intradural-extramedullary spinal cord tumors. We suggest that this technique is one of the best treatments for these tumors.

KEY WORDS : Laminectomy · Intradural extramedullary spinal cord neoplams

\title{
INTRODUCTION
}

Spinal cord tumors occur rarely, at an incidence of 10 per 100,000 people $^{14)}$. The most common spinal cord tumors are nerve sheath tumors, such as schwannoma and neurofibroma $(30 \%)$, which are followed by meningioma (25\%) and others, like astrocytoma and angioblastoma ${ }^{7}$.

Spinal cord tumors have usually been removed through total laminectomy, and this method is still used widely ${ }^{3)}$. Seppala et al. ${ }^{12}$ analyzed 187 patients who had total laminectomy for spinal cord tumors and reported satisfactory prognoses. However, total laminectomy may cause spinal instability and kyphosis due to the damage to the musculoligamentous structures and posterior bony elements. And these complications may produce neurologic symptoms by compressing the spinal cord or nerve roots ${ }^{17)}$. In order to prevent such complications, a total laminectomy with arthrodesis or a unilateral limited laminectomy, which was reported to be useful by Yasargil et al ${ }^{16}{ }^{16}$ in 1991, often has to be performed. In this study, we describe our experiences in the use of unilateral limited laminectomy to intradural-extramudullary spinal cord tumors, along with a review of the literature.

Table 1. Details of patients who underwent the unilateral limited laminectomy for a spinal cord tumor

\footnotetext{
- Received : December 7, 2006

- Accepted : May 1, 2008

- Address for reprints :

Hyung-Dong Kim, M.D.

Department of Neurosurgery

College of Medicine

Dong-A University

Dongdaesin-dong 3-ga

Seo-gu, Busan 602-715, Korea

Tel : $+82-51-240-5240$

Fax : +82-51-242-6714

E-mail : hdkim@donga.ac.kr
}

\begin{tabular}{|c|c|c|c|c|c|}
\hline Patient No. & Age/Sex & Location & Size $(\mathrm{mm})$ & Side & Histopathology \\
\hline 1 & 58/Female & Cervicomedullary & $18 \times 17 \times 23$ & Right, Ventral & Schwannoma \\
\hline 2 & 19/Female & Cervical 4-5 & $14 \times 10 \times 15$ & Right, Ventral & Schwannoma \\
\hline 3 & 66/Male & Cervical 5-6 & $11 \times 9 \times 13$ & Right, Ventral & Schwannoma \\
\hline 4 & 83/Male & Cervical 6-7 & $11 \times 9 \times 13$ & Right, Dorsal & $\begin{array}{c}\text { Meningioma } \\
\text { (Meningothelial) }\end{array}$ \\
\hline 5 & 65/Male & Thoracic 11-12 & $15 \times 13 \times 39$ & Left, Dorsal & Schwannoma \\
\hline 6 & 24/Male & $\begin{array}{c}\text { Thoracolumbar } \\
\text { T12-L1 }\end{array}$ & $17 \times 17 \times 27$ & $\begin{array}{c}\text { Central to left, } \\
\text { Ventral }\end{array}$ & Schwannoma \\
\hline 7 & 61/Female & Lumbar 1 & $13 \times 12 \times 25$ & Right, Dorsal & Schwannoma \\
\hline
\end{tabular}



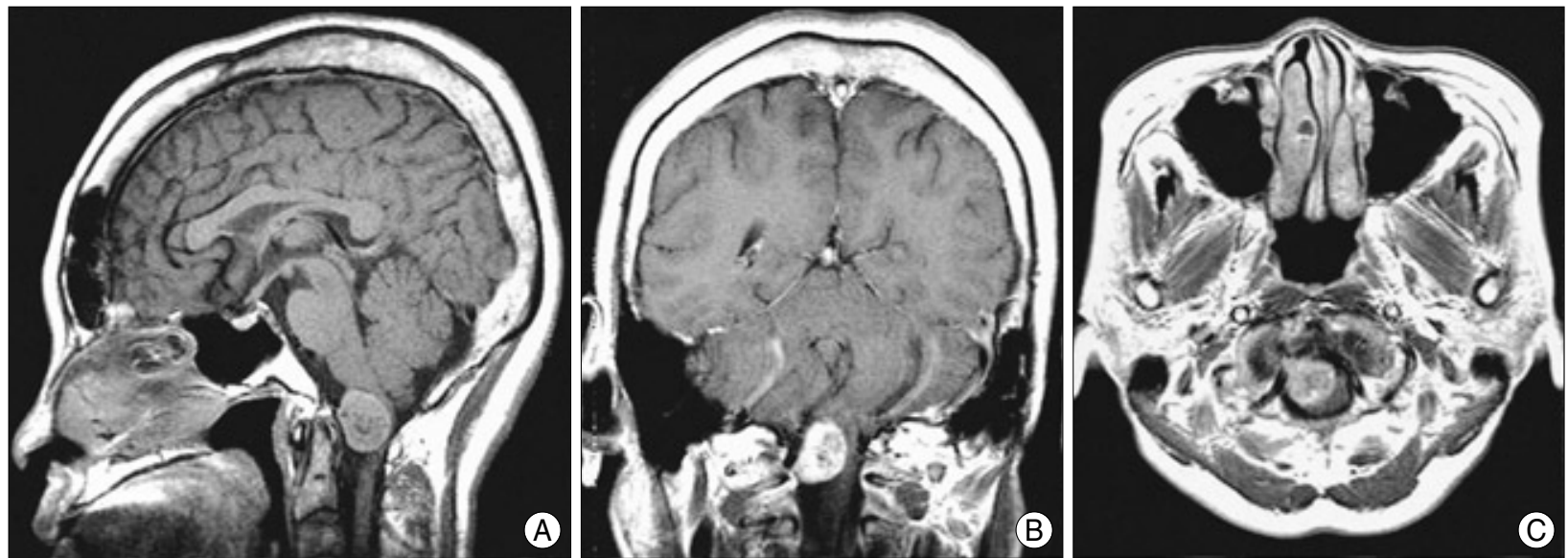

Fig. 1. Case 1. In preoperative gadolinium enhanced T1-weighted sagittal (A), coronal (B), and axial (C) MRI scans, an $18 \times 17 \times 23 \mathrm{~mm}$ sized inhomogeneous signal intensity mass was seen in the cranio-vertebral junction. This mass was located primarily on the right side and it compressed and displaced the medulla to the left side.
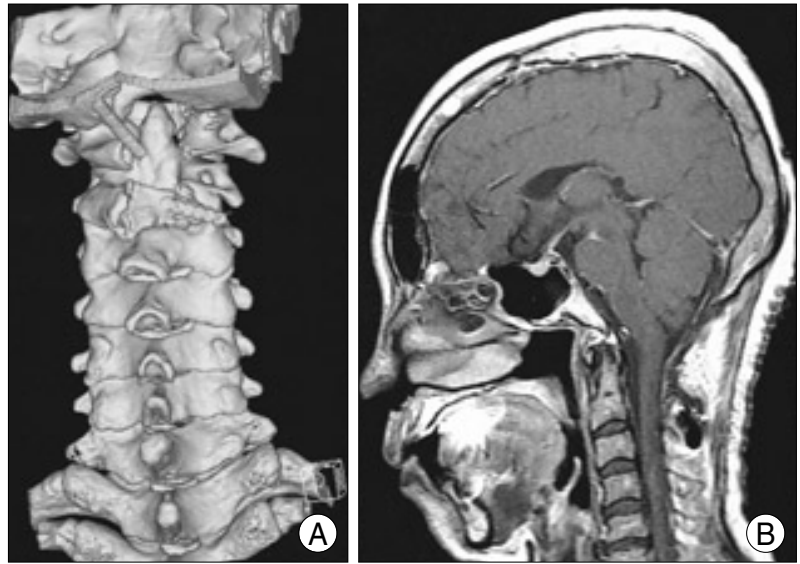

Fig. 2. Case 1. Postoperative 3D CT scan, hemilaminectomy of $\mathrm{C} 1$ and laminoplasty of $\mathrm{C} 2$ with miniplate was shown (A). A postoperative gadolinium enhanced T1-weighted sagittal MRI scan showed that the tumor was totally removed (B).

\section{CASE REPORT}

We performed seven unilateral laminectomies for intradural-extramedullary spinal cord tumors from March 2006 to December 2006 (Table 1).

In the cervical case (Case 1), a 58-year-old female presented with a 3-month history of vertigo. On examination, there was dysesthesia along the dermatomes of $\mathrm{C} 1$ and $\mathrm{C} 2$ on the right side when the neck was flexed. The magnetic resonance imaging (MRI) of the cervical spine revealed a well-enhanced $18 \times 17 \times 23 \mathrm{~mm}$-sized mass at the right side of the cranio-vertebral junction (Fig. 1). The mass was predicted to be schwannoma. Based on examination and image study, we decided to approach by right unilateral laminectomy.

Under general anesthesia, the patient was placed prone in pin fixation. A midline skin incision was made from the
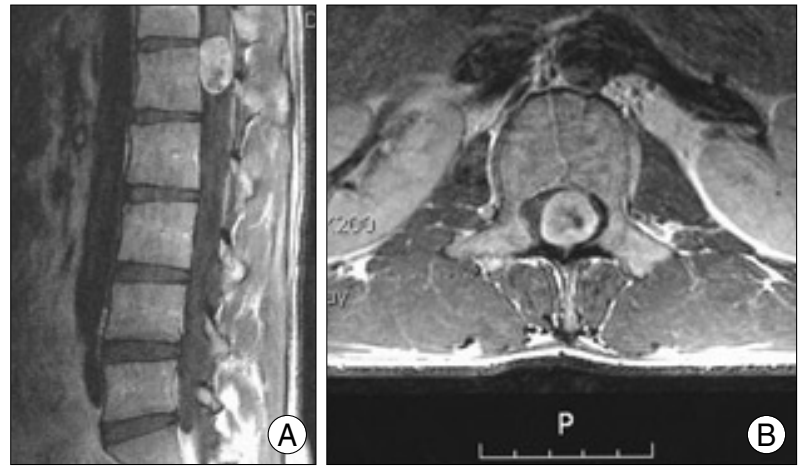

Fig. 3. Case 6. In preoperative gadolinium enhanced T1-weighted sagittal (A) and axial (B) MRI scans, a $17 \times 17 \times 27 \mathrm{~mm}$ sized well-defined mass was seen in the Conus medullaris. This mass showed wellenhancement signal intensity and a hypointensity signal on the central portion.

inion to the spinous process of $\mathrm{C} 4$. The unilateral occipital bone and neural arches of $\mathrm{C} 1,2$ were exposed on the right side only. Under microscopic magnification, a unilateral limited laminectomy was performed on the right side lamina of $\mathrm{C} 1$ and the right occipital bone by high-speed drill and kerrison punch. The dura was then opened paramedially. The tumor was located at the ventrolateral side of the medulla oblongata. After the tumor was found, the affected root was localized. For more space, laminoplasty was performed on the right side lamina of $\mathrm{C} 2$. After careful separation of the $\mathrm{C} 1$ ventral root and sectioning of the fibers entering the mass, the tumor was removed completely without any difficulty (Fig. 2). The dura was closed with prolene 8-0 suture to obtain a waterproof closure. Fibrin glue was then instilled to cover the closed dura surface. The fascia was reattached to the spinous processes of $\mathrm{C} 1-\mathrm{C} 4$, and the remainder of the wound was closed in anatomic layers. Schwannoma was confirmed on histopathology.

In the lumbar case (Case 6), a 24-year-old male presented 

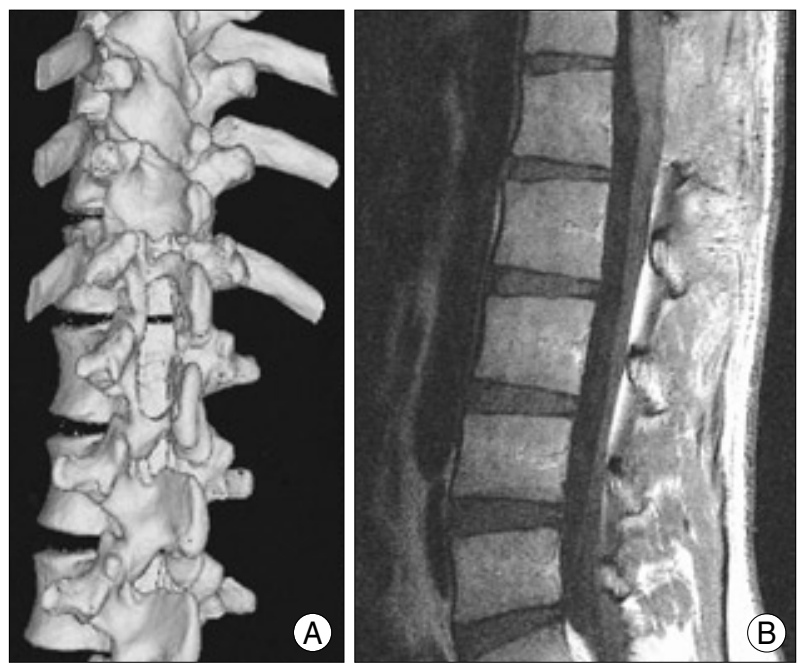

Fig. 4. Case 6. In a postoperative 3D CT scan, the hemilaminectomies of $\mathrm{T} 12$ and $\mathrm{L} 1$ were shown $(\mathrm{A})$. In a postoperative gadolinium enhanced T1-weighted sagittal MRI scan, the tumor was totally removed (B).

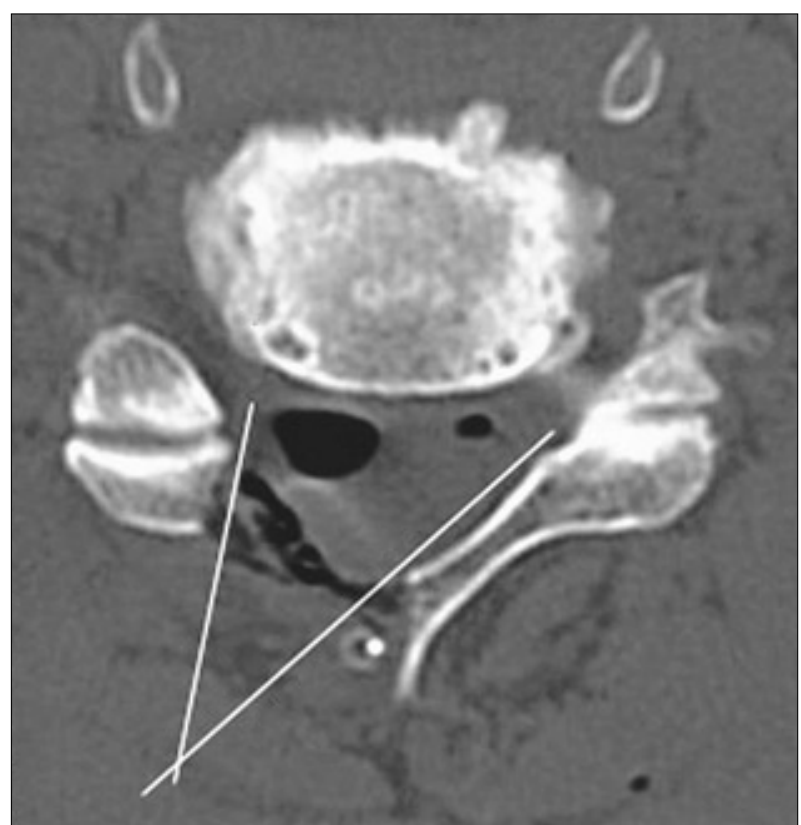

Fig. 5. Hemilaminectomy was performed and the base of the spinous processes was undercut to gain access to the contralateral side lesion.

with a 1-month history of radiating pain in the left thigh. On examination, there was dysesthesia along the dermatomes of L1 and L2 of the left thigh, and the left deep tendon reflexes were increased slightly compared to the right. The MRI of the lumbar spine revealed a well-enhanced $17 \times$ $17 \times 27 \mathrm{~mm}$-sized mass at the 12th thoracic and the 1st lumbar vertebrae (Fig. 3). The mass was predicted to be schwannoma. Based on symptom and image study, we decided to approach by left unilateral laminectomy.

A midline skin incision was made from $\mathrm{T} 11$ to the $\mathrm{L} 2$ spinous process tip. The paravertebral muscles were detached on the left side only in the same way as in the previous case. A unilateral limited laminectomy was performed on the left side laminae of T12, L1. The dura was opened paramedially. The tumor was located at the dorsolateral side of the conus medullaris. After careful separation of the T12 dorsal root and sectioning of the fibers entering the mass, the tumor was removed completely without any difficulty (Fig. 4). The wound was closed in anatomic layers as in the previous case. Schwannoma was confirmed on histopathology.

For more space for the removal of spinal cord tumors, the base of the spinous process was undercut to permit progressive contralateral visualization with table tilting. And we did tried not to disrupt the facet joint. The surgical exposure through this corridor was satisfactory for spinal cord tumor removal (Fig. 5). But in the step of dural closure, it is somewhat difficult to close the dura matter because the space for the needle working was a little narrow. So we used a small needle, like a prolene $8-0$.

The locations and histopathological findings of our cases are shown in Table 1. The masses were located in the cervical area in four cases, in the thoracolumbar region in two cases, and in the lumbar region in one case. According to the histopathology, Case 4 was diagnosed as meningioma (meningothelial type) and the others as schwannoma.

In most of the cases, lesions were exposed with a two level hemilaminectomy, but in two cases, a three level hemilaminectomy was performed. In all cases, transfusion was not necessary during the operation.

All of the patients were ambulated on the third postoperative day and could be discharged within 10 days without any neurological deficits or complications postoperatively. No external orthotics were used for any of the patients. The patients with preoperative neurological deficits recovered in a few weeks and there were no neurological deficits at the last follow-up. At follow-up evaluation, the patients showed normal alignment and no instability in the post-operative period (at 3 and 12 months after the operation, mean 18 months period).

\section{DISCUSSION}

Traditionally, the surgical resection of a spinal cord tumor has been performed through total laminectomy ${ }^{3}$. This facilitates access and visualization. Sepalla et al. ${ }^{12)}$ reported a series of 187 patients that underwent surgical resection for spinal schwannoma. In this series, $90 \%$ were completely resected, with a $10 \%$ surgical complication rate and $1.5 \%$ surgical fatality rate. Levy et al. ${ }^{6}$ also reported similar results for 66 patients with a $9 \%$ surgical complication rate and a $1.5 \%$ mortality rate. 
However, it has been reported by a number of researchers that total laminectomy may bring several complications. According to their reports, the most common complication was kyphosis ${ }^{1,4,10,11)}$. Bilateral damage to the ligamentum flavum and disruption of the ligamentum interspinosum were considered to play an important role ${ }^{4)}$. Stripping, dissection, and denervation of the posterior paraspinal muscle complex were also suggested to be responsible for post-laminectomy deformities ${ }^{1,11)}$. Post-laminectomy kyphosis may occur within weeks to years after a laminectomy for a tumor or trauma, especially in children that have not reached bone maturity ${ }^{1,10)}$. Another reported complication was progressive myelopathy, which was preceded by other problems, including spinal deformity, instability, epidural fibrosis, and the absence of osseous protection for the spinal cord $^{17)}$. The other reported probable complications were cerebrospinal fluid leak and wound infection ${ }^{8)}$.

Therefore it has been recommended that the spine be fused, primarily at the time of the initial decompressive laminectomy, to reduce the risk of postoperative spinal instability and deformity ${ }^{1,13)}$. A minimal invasive technique has also been emphasized for spinal surgery, rather than an extensive destructive technique. Unilateral laminectomy was reported at the beginning of spinal surgery and was popularized by Eggert et al. in the 1980's with the advance of operating microscopes ${ }^{4,5)}$. In 1991, Yasargil et al. ${ }^{16)}$ suggested unilateral laminectomy for intradural tumors, and Oktem et al. ${ }^{8)}$ described their experience with 20 patients undergoing unilateral hemilaminectomy for intradural tumor resection. Of these patients, none exhibited spinal instability after two years of follow-up.

The advantages of a unilateral laminectomy are that it reduces posteroperative pain, prevents instability, avoids the use of external bracing, and allows early mobilization of the patient ${ }^{9}$. In addition, unilateral laminectomy has been associated with less blood loss, better wound healing, less risk of postoperative infection, and shorter hospital stays than traditional total laminectomy $y^{2,15)}$.

It may be assumed that the view is narrow in a unilateral laminectomy, so it is not easy to handle instruments and the exposure of the lesion is incomplete, and as a result, normal nerves may be damaged or it may be difficult to remove the lesion completely. So unilateral drilling may be extended to the facets which, when necessary, can be partially resected. This provides a more lateral visualization and allows a greater opportunity for anterior maneuvering than exposure with a standard laminectomy ${ }^{2}$. For tumors that were not extended to the vertebral foramen, there was no need to do a facetectomy. In our experience, without a facetectomy, the view was sufficient for the operation and the lesion was clearly identified and fully exposed (Fig. 5). Dural reapproximation was also not difficult. A small needle, like a prolene $8-0$, was used for simple manual knot tying and running sutures.

Sarioglu et al. ${ }^{11)}$ reported their experience with 40 spinal tumors that were removed by unilateral hemilaminectomy. They concluded that the unilateral laminectomy could be applied to all spinal tumors, except bilateral extensive invading extradural tumors, with the aid of microneurosurgical techniques. Some authors have considered that an intradural tumor could be removed unilaterally even if it was situated in the ventral to the cord or midline lesion ${ }^{4,15)}$. But we do not include tumors, which are located on the ventral to the spinal cord or extended to the vertebral foramen, and also intramedullary spinal cord tumors as indication.

Our study group is too small and the follow-up period is too short to make a definite statement with respect to the applicability of the unilateral approach to intraduralextramedullary spinal cord tumors. But the results from this small series represent our experiences in the use of unilateral limited laminectomy to intradural-extramedullary spinal cord tumors.

\section{CONCLUSION}

Intradural-extramedullary spinal cord tumors that are not extended to the vertebral foramen can be resected safely and completely by a unilateral limited laminectomy. Although this small series of consecutive patients with a short follow-up, we have demonstrated that it is possible to completely resect intradural-extramedullary spinal cord tumors safely with the reductions in postoperative back pain, instability, degenerative changes and operative blood loss.

\section{References}

1. Alexander E Jr : Post-laminectomy kyphosis in Wilkins RH, Rengachary SS(eds) : Neurosurgery. New York : McGraw-Hill, 1985, pp 2293-2297

2. Bertalanffy H, Mitani S, Otani M, Ichikizaki K, Toya S : Usefullness of hemilaminectomy for microsurgical management of intraspinal lesions. Keio J Med 41 : 76-79, 1992

3. Cervoni L, Celli P, Cantore G, Fortuna A : Intradural tumors of the cauda equina : a single institution review of clinical characteristics. Clin Neurol Neurosurg $97: 8-12,1995$

4. Chiou SM, Eggert HR, Laborde G, Seeger W : Microsurgical unilateral approaches for spinal tumour surgery : eight years' experience in 256 primary operated patients. Acta Neurochir (Wien) 100 : 127-133, 1989

5. Eggert HR, Scheremet R, Seeger W, Gaitzsch J : Unilateral microsurgical approaches to extramedullary spinal tumours. Operative technique and results. Acta Neurochir (Wien) 67 : 245-253, 1983

6. Levy WJ, Latchaw J, Hahn JF, Sawhny B, Bay J, Dohn DF : Spinal neurofibromas : a report of 66 cases and a comparison with meningiomas. Neurosurgery $18: 331-334,1986$

7. Masaryk TJ : Neoplastic disease of the spine. Radiol Clin North Am 29 : 829-845, 1991 
8. Oktem IS, Akdemir H, Kurtsoy A, Koc RK, Menku A, Tucer B : Hemilaminectomy for the removal of the spinal lesions. Spinal Cord $38: 92-96,2000$

9. Pompili A, Caroli F, Cattani F, Crecco M, Giovannetti M, Raus L, et al : Unilateral limited laminectomy as the approach of choice for the removal of thoracolumbar neurofibromas. Spine $29: 1698-1702$, 2004

10. Reimer R, Onofrio BM : Astrocytomas of the spinal cord in children and adolescents. J Neurosurg 63: 669-675, 1985

11. Sarioglu AC, Hanci M, Bozkus H, Kaynar MY, Kafadar A : Unilateral hemilaminectomy for the removal of the spinal space-occupying lesions. Minim Invasive Neurosurg 40 : 74-77, 1997

12. Seppala MT, Haltia MJ, Sankila RJ, Jaaskelainen JE, Heiskanen O : Long-term outcome after removal of spinal schwannoma : a clinicopathological study of 187 cases. J Neurosurg 83 : 621-626, 1995
13. Simeone FA : Spinal cord tumors in adults in Youmans JR (eds) : Neurological Surgery. Philadelphia : WB Saunders Company, 1990, pp 3531-3547

14. Stein BM, McCormick PC : Intramedullary neoplasms and vascular malformations. Clin Neurosurg 39 : 361-387, 1992

15. Tredway TL, Santiago P, Hrubes MR, Song JK, Christie SD, Fessler RG : Minimally invasive resection of intradural-extradullary spinal neoplasms. Neurosurgery 58 (1 suppl) :52-58, 2006

16. Yasargil MG, Tranmer BI, Adamson TE, Roth P : Unilateral partial hemi-laminectomy for the removal of extra- and intramedullary tumours and AVMs. Adv Tech Stand Neurosurg : 113-132, 1991

17. Yasuoka S, Peterson HA, MacCarty CS : Incidence of spinal column deformity after multilevel laminectomy in children and adults. J Neurosurg $57: 441-445,1982$ 